\title{
Extração e quantificação de carotenoides em minitomate desidratado (Sweet Grape) através da aplicação de diferentes solventes
}

\author{
Extraction and quantification of carotenoids from dehydrated mini-tomatoes \\ (Sweet Grape) by applying different solvents
}

\author{
Stéfani Cristina Nellis ${ }^{1 *}$, Angela de Fátima Kanesaki Correia' ${ }^{1}$, Marta Helena Fillet Spoto ${ }^{2}$ \\ 'Universidade Metodista de Piracicaba (UNIMEP), Faculdade de Engenharia, Arquitetura e Urbanismo, Santa Bárbara d'Oeste/SP - Brasil \\ ¿Universidade de São Paulo (USP), Escola Superior de Agricultura "Luiz de Queiroz" (ESALQ), Departamento de Agroindustria, Alimentos e Nutrição \\ (LAN), Piracicaba/SP - Brasil
}

\section{*Corresponding Author}

Stéfani Cristina Nellis, Universidade Metodista de Piracicaba (UNIMEP), Faculdade de Engenharia, Arquitetura e Urbanismo, Rodovia Luís Ometto, km 24 (SP-306), CEP: 13451-900, Santa Bárbara d'Oeste/SP - Brasil, e-mail: stefaninellis@gmail.com

Cite as: Extraction and quantification of carotenoids from dehydrated mini-tomatoes (Sweet Grape) by applying different solvents. Braz. J. Food Technol., v. 20, e2016156, 2017.

Received: Oct. 26, 2016; Accepted: Apr. 04, 2017

\section{Resumo}

Atualmente, devido a eventos como a urbanização, globalização e industrialização, houve um grande aumento de doenças ligadas à alimentação. Acredita-se, em grande parte, que o consumo de frutas e hortaliças auxilia na prevenção de muitas dessas doenças. O tomate contém alto teor de carotenoides, que são pigmentos vermelho-alaranjados presentes e distribuídos em algumas hortaliças. No caso do tomate, são encontrados, principalmente, os carotenoides licopeno e betacaroteno. O primeiro é encontrado em maior quantidade e age como um importante antioxidante, enquanto o segundo é encontrado em menor quantidade no fruto, apresentando atividade provitamina A. Devido a essas e várias outras funções desempenhadas pelos carotenoides, eles vêm sendo determinados há muito tempo em vários alimentos. São extraídos facilmente com solventes orgânicos, mas apresentam instabilidade diante de agentes como luz, oxigênio e aquecimento. Sendo assim, o presente trabalho teve como objetivo analisar o processo de extração e quantificação de carotenoides do tomate cereja desidratado da variedade Sweet Grape por meio da combinação de solventes: acetona/éter de petróleo, acetona/clorofórmio e acetona/hexano, possibilitando verificar o melhor solvente para o procedimento. Os carotenoides foram quantificados em espectrofotômetro com leitura de $350 \mathrm{~nm}$ a $600 \mathrm{~nm}$, referindo-se aos picos de absorção do betacaroteno e licopeno. Os resultados obtidos mostraram que o solvente com maior desempenho para extração de carotenoides no produto em questão foi a combinação de acetona/hexano, seguida da de acetona/éter de petróleo e acetona/clorofórmio. A última combinação exigiu um procedimento operacional de extração mais difícil. Com a combinação de acetona/hexano

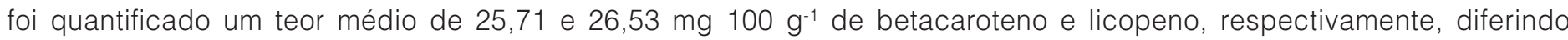
estatisticamente, ao nível de $5 \%$ de significância, da extração com os demais solventes.

Palavras-chave: Extração; Quantificação; Licopeno; Betacaroteno; Minitomates; Solventes.

\section{Abstract}

Nowadays, due to events such as urbanization, globalization and industrialization there has been an increase in illnesses connected to eating. It is believed that, in part, the consumption of fruits and vegetables helps prevent many of these diseases. Tomatoes contain high contents of carotenoids, which are red-orange pigments present in some vegetables. In the case of tomatoes, the carotenoids mostly found are lycopene and beta-carotene. Lycopene is found in greater amounts and acts as an important antioxidant, whereas beta-carotene is found in lesser amounts in the fruit and presents pro-vitamin A activity. Due to these and various other functions carried out by the carotenoids, they have been determined for a long time in various foods. They are easily extracted with organic solvents but show instability when in the presence of agents such as light, oxygen and heat. Thus this study aimed to analyze the extraction and quantification processes of carotenoids from dehydrated mini-tomatoes of the variety Sweet Grape by means of the solvent combinations: acetone/petroleum ether, acetone/chloroform 
and acetone/hexane, in order to verify which solvent was the best for this procedure. The carotenoids were quantified using a spectrophotometer by reading at 350-600 nm, referring to the absorption peaks of beta-carotene and lycopene. The results showed that the solvent with the best performance for extraction of the carotenoids from the product in question was the combination of acetone and hexane, followed by acetone/petroleum ether and finally acetone/chloroform, the latter also presenting a more difficult operational extraction procedure. With the acetone/hexane combination average contents of 25.71 and $26.53 \mathrm{mg} 100 \mathrm{~g}^{-1}$ beta-carotene and lycopene, respectively, were extracted, showing a statistically significant difference at the $5 \%$ level from the extractions using the other solvents.

Keywords: Extraction; Quantification; Lycopene; Beta-carotene; Tomatoes; Solvents.

\section{Introdução}

A relação entre alimentação e saúde é um assunto muito relevante, já que eventos como a urbanização, industrialização e globalização influíram no estado nutricional das pessoas. O aumento das doenças ligadas à dieta, como obesidade, diabetes, problemas cardiovasculares, hipertensão e câncer está ligado diretamente à má alimentação dos indivíduos. Acredita-se que o consumo de frutas e hortaliças auxilia, em grande parte, na prevenção destas doenças (CARVALHO et al., 2006).

Um crescimento muito grande na horticultura tem sido observado no Brasil, principalmente no desenvolvimento de pesquisas visando ao melhoramento genético em sementes como as do tomate. Entre os tipos de tomate lançados recentemente no mercado, destacam-se os tomates tipo cereja, que possuem cor vermelho intenso, firmeza e um valor nutricional que difere quando comparado a de outros cultivares (AGUIAR et al., 2012).

Um dos híbridos que mais vem se destacando é o tomate "Sweet Grape", que foi desenvolvido pela empresa japonesa Sakata, do segmento de sementes, e que possui características como: tamanho pequeno com formato alongado, coloração vermelho intenso, baixo índice de acidez, teor de açúcar elevado, entre outros (JUNQUEIRA et al., 2011).

O tomate é uma hortaliça com grande teor de carotenoides, que podem ser definidos como uma classe de pigmentos amarelo-alaranjado-vermelhos, que se encontram distribuídos em várias frutas e hortaliças. Alguns carotenoides são precursores de vitamina A, uma função muito importante já que a sua deficiência pode acarretar problema de saúde pública. No tomate, o betacaroteno é o carotenoide provitamina A mais ativo, e que está associado à proteção contra doenças cardíacas e câncer (CARVALHO et al., 2006).

O licopeno é outro carotenoide encontrado em elevada concentração no tomate e seus produtos derivados, possuindo uma importante função antioxidante. Além disso, a presença de licopeno na dieta se relaciona com a redução de certos tipos de câncer e redução do risco de ataque cardíaco (CARVALHO et al., 2006).

A presença de antioxidantes nos alimentos pode explicar o poder de curar e prevenir muitos dos nossos problemas de saúde. Os oxidantes se formam através da ação de formas tóxicas do oxigênio, podendo atuar na obstrução das artérias, na transformação das células em cancerosas, ocasionar problemas nas articulações, além de estar associadas ao envelhecimento. Os oxidantes assumem várias formas e aspectos, sendo que a mais comum é a dos radicais livres. Sendo assim, os antioxidantes atuam combatendo a formação e ação dos radicais livres (CARVALHO et al., 2006).

Existem métodos biológicos, químicos e físicos para avaliação dos carotenoides. Entretanto, a sua determinação pode ser dificultada devido à sua instabilidade diante de agentes como o oxigênio, luz e aquecimento. Já a separação dos carotenoides é facilmente conseguida se fazendo uso de solventes orgânicos (MORAES et al., 1974).

Devido às suas relevantes funções biológicas no ser humano, os carotenoides vêm sendo determinados desde longa data nos mais diferentes tipos de alimentos, aliados também ao seu importante papel como pigmentos naturais (WILBERG; RODRIGUEZ-AMAYA, 1993).

Desse modo, visto a importância dos carotenoides para a alimentação e para o setor alimentício, o presente trabalho teve por objetivo analisar o processo de extração e quantificação de carotenoides no tomate cereja desidratado da variedade Sweet Grape por meios de diferentes solventes, a fim de comparar e identificar o melhor solvente para extração e quantificação deste pigmento.

\section{Material e métodos}

\subsection{Amostra de minitomate desidratado Sweet Grape}

As amostras de minitomates desidratados da variedade Sweet Grape foram fornecidas por um projeto de pesquisa do Laboratório de Frutas e Hortaliças do Departamento de Agroindústria, Alimentos e Nutrição (LAN/ESALQ), localizado em Piracicaba, Estado de São Paulo. As amostras recebidas haviam passado previamente pelas etapas de seleção, lavagem, sanitização, envase, congelamento, desidratação osmótica, desidratação adiabática e foram embaladas e mantidas refrigeradas a $5{ }^{\circ} \mathrm{C}$ até o momento de realização das análises.

$\mathrm{Na}$ etapa de seleção, os minitomates foram visualmente selecionados com relação à aparência, uniformidade, coloração, grau de maturação e ausência 
Extração e quantificação de carotenoides em minitomate desidratado (Sweet Grape) através da aplicação de diferentes solventes Nellis, S. C. et al.

de danos físicos. Em seguida, foram higienizados em água corrente a fim de remover sujidades superficiais e sanitizados através da imersão em solução de cloro a $200 \mathrm{mg} \mathrm{L}^{-1}$ durante 15 minutos.

As amostras foram embaladas em embalagens flexíveis de polietileno de baixa densidade fechadas em seladora Tec-Maq, modelo AP 450, sendo, em seguida, dispostas em prateleiras da câmara de congelamento marca Schmidt STR-6, a $-22^{\circ} \mathrm{C} \pm 2{ }^{\circ} \mathrm{C}$.

Depois de embaladas, as amostras de minitomate passaram por um pré-tratamento osmótico em solução composta por açúcar invertido (60 Brix), sacarose (15\%) e cloreto de sódio (5\%), à temperatura de $25{ }^{\circ} \mathrm{C}$ por 50 minutos.

As amostras foram submetidas à desidratação adiabática em duplo estágio, $80^{\circ} \mathrm{C}$ durante 2 horas e $70^{\circ} \mathrm{C}$ durante 11 horas. Depois da desidratação adiabática, as amostras foram acondicionadas em embalagem laminada de poliéster metalizado, adesivo e polietileno e mantidas sob refrigeração a $5^{\circ} \mathrm{C} \pm 1{ }^{\circ} \mathrm{C}$, até o momento da realização das análises.

\subsection{Extração dos carotenoides}

No procedimento de extração de carotenoides das amostras de minitomate desidratado, foram utilizadas as seguintes combinações de solventes: acetona/éter de petróleo, acetona/clorofórmio e acetona/hexano. A acetona foi utilizada para extração inicial dos carotenoides na amostra.

A análise de carotenoides consiste, geralmente, na amostragem e preparação das amostras, extração e partição com um solvente, saponificação e lavagem, concentração ou evaporação do solvente, separação cromatográfica e identificação e quantificação em espectrofotômetro.

Desse modo, a extração dos carotenoides foi realizada segundo Rodriguez-Amaya (2001). Como as amostras estavam desidratadas, foi necessário que, primeiramente, fossem hidratadas, utilizando-se água destilada na proporção 1:3 e, em seguida, homogeneizadas. Posteriormente, foi pesado $1 \mathrm{~g}$ das amostras em balança analítica, que foi transferido para um graal de vidro adicionado de $10 \mathrm{~g}$ de celite. O solvente utilizado para a extração inicial dos carotenoides foi $30 \mathrm{~mL}$ de acetona. Em seguida, foi realizada a maceração e a mistura obtida foi filtrada a vácuo em funil de buchner para um kitassato de $250 \mathrm{~mL}$. O extrato cetônico do kitassato foi transferido para um funil de separação contendo, aproximadamente, $20 \mathrm{~mL}$ do segundo solvente. Para a remoção total da acetona e transferência dos carotenoides para o segundo solvente, foram feitas lavagens com água destilada por três vezes, sendo que na última lavagem foi adicionado sulfato de sódio anidro diretamente no funil de separação, que auxiliou na separação das fases. Depois das lavagens, ocorreu a separação do extrato (fase superior) e água (fase inferior) no funil de separação, sendo essa descartada.

O extrato foi transferido, então, para um balão volumétrico de $25 \mathrm{~mL}$ e avolumado com o segundo solvente. Em seguida, foi realizada a varredura em espectrofotômetro no intervalo de 350 a $600 \mathrm{~nm}$, que se refere aos picos de absorção do licopeno e betacaroteno.

Para a extração dos carotenoides com os solventes, a leitura da absorbância no espectro foi feita usando o segundo solvente como "branco". Todos os procedimentos foram realizados em triplicata e com a devida proteção contra a luz, a fim de evitar a perda de compostos.

O processo experimental da extração dos carotenoides é representado na Figura 1 em forma de fluxograma.

\subsection{Determinação do teor de carotenoides}

A determinação do teor de carotenoides das amostras foi realizada em espectrofotômetro UV-Vis - FEMTO modelo 800 XI, conforme metodologia analítica de separação e extração dos compostos com solventes orgânicos. Para determinação do licopeno, foi utilizada a absorbância lida em 470 nm e, para o betacaroteno, a absorbância lida em $450 \mathrm{~nm}$. Desse modo, os carotenoides foram determinados de acordo com a Equação 1 (RODRIGUEZ-AMAYA, 2001; RODRIGUEZ-AMAYA; KIMURA, 2004).

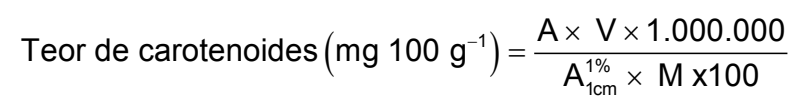

onde: $A$ = absorbância da solução no comprimento de onda de $470 \mathrm{~nm}$ para o licopeno e de $450 \mathrm{~nm}$ para o betacaroteno; $V$ = volume final da solução; $A_{1 \mathrm{~cm}}^{1 \%}=$ coeficiente

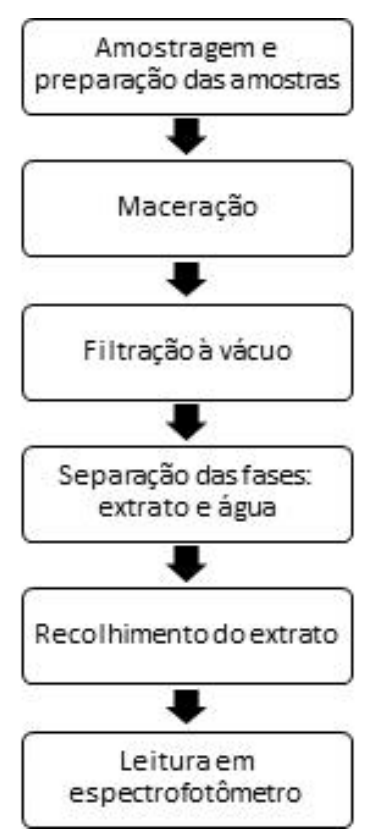

Figura 1. Fluxograma de extração de carotenoides dos minitomates desidratados variedade Sweet Grape. 
Extração e quantificação de carotenoides em minitomate desidratado (Sweet Grape) através da aplicação de diferentes solventes Nellis, S. C. et al.

de extinção ou coeficiente de absortividade molar de um pigmento em um determinado solvente específico (3450 para o licopeno e 2592 para o betacaroteno); M = massa da amostra tomada para a análise.

\section{Resultados e discussão}

Na Tabela 1, são apresentados os resultados obtidos referentes à concentração média do pigmento beta caroteno do minitomate desidratado da variedade Sweet Grape, através dos diferentes solventes utilizados. Observou-se que a combinação de acetona e hexano apresentou maior capacidade extratora, seguida dos solventes acetona/éter de petróleo e acetona/clorofórmio. Além disso, o hexano diferiu significativamente, ao nível de 5\% de significância, dos demais solventes utilizados, indicando ter maior desempenho nas amostras de tomate desidratado. Entre os solventes éter de petróleo e clorofórmio, a capacidade extratora se mostrou próxima, e não diferiu significativamente entre ambas ao nível de significância de 5\%.

$\mathrm{Na}$ Tabela 2, são apresentados os resultados obtidos referentes à concentração média de licopeno nas amostras de minitomate desidratado, através dos solventes utilizados. Constatou-se, novamente, a eficiência da combinação de acetona e hexano na capacidade extratora do pigmento carotenoide licopeno. O hexano foi o solvente mais eficiente nas amostras desidratadas e diferiu significativamente $(p<0,05)$ dos resultados obtidos com os demais solventes. Na sequência, encontram-se os solventes acetona/éter de petróleo e acetona/clorofórmio, sendo o último o de pior desempenho. Além disso, a

Tabela 1. Teor de betacaroteno em minitomate desidratado (Sweet Grape) através dos solventes para extração: éter de petróleo, clorofórmio e hexano.

\begin{tabular}{lc}
\multicolumn{1}{c}{ Solvente } & $\begin{array}{c}\text { Concentração média } \\
\left(\mathbf{m g} \mathbf{1 0 0} \mathbf{~ g}^{-\mathbf{1}} \mathbf{)}\right.\end{array}$ \\
\hline Acetona/Éter de petróleo & $21,14^{\mathrm{a}} \pm 0,96$ \\
Acetona/Clorofórmio & $20,15^{\mathrm{a}} \pm 1,85$ \\
Acetona/Hexano & $25,71^{\mathrm{b}} \pm 0,87$ \\
\hline
\end{tabular}

Nota: Resultados expressos pela média \pm desvio padrão. Em uma mesma coluna, média seguida de letras iguais indica que as amostras não diferem entre si pelo teste de Tukey, ao nível de significância de 5\%.

Tabela 2. Teor de licopeno em minitomate desidratado (Sweet Grape) através dos solventes para extração: éter de petróleo, clorofórmio e hexano.

\begin{tabular}{lc}
\multicolumn{1}{c}{ Solvente } & $\begin{array}{c}\text { Concentração média } \\
\left(\mathbf{m g ~} \mathbf{0 0} \mathbf{~ g}^{-1}\right)\end{array}$ \\
\hline Acetona/Éter de petróleo & $19,85^{\mathrm{a}} \pm 0,77$ \\
Acetona/Clorofórmio & $16,33^{\mathrm{b}} \pm 0,69$ \\
Acetona/Hexano & $26,53^{\mathrm{c}} \pm 1,72$ \\
\hline
\end{tabular}

Nota: Resultados expressos pela média \pm desvio padrão. Em uma mesma coluna, média seguida de letras iguais indica que as amostras não diferem entre si pelo teste de Tukey, ao nível de significância de 5\%. extração com o solvente clorofórmio mostrou-se mais difícil, já que as fases (extrato e água) se separavam de modo diferente das demais amostras, interferindo no desempenho e dificultando o procedimento de extração.

De acordo com Stahl e Sies (1999), o teor de licopeno em tomates vermelhos maduros é $30-80 \mu \mathrm{g} \mathrm{g}^{-1}$ de produto fresco, podendo ser mais do que 150 a $300 \mu \mathrm{g} \mathrm{g}^{-1} \mathrm{em}$ espécies selecionadas obtidas por técnicas de hibridização tradicional.

Em um estudo realizado por Lima et al. (2002), que utilizou uma metodologia semelhante a este trabalho, foi encontrado no tomate in natura um teor de carotenoides totais equivalente a $54,5 \mathrm{\mu g} \mathrm{g}^{-1}$, no qual a quantidade do licopeno corresponde a $42,85 \mu \mathrm{g} \mathrm{g}^{-1}$, já que é o pigmento carotenoide encontrado em maior quantidade no fruto.

A Figura 2 apresenta o gráfico das leituras obtidas no espectrofotômetro, correspondente ao comprimento de onda utilizado em função da absorbância das amostras, ao se utilizar os solventes acetona/hexano, acetona/clorofórmio e acetona/éter de petróleo. Foi feita a varredura no comprimento de onda entre $350 \mathrm{~nm}$ e $600 \mathrm{~nm}$, intervalo que apresenta o pico de absorção do licopeno e betacaroteno, que posteriormente foi utilizado para quantificação. Foi perceptível a diferença da combinação de acetona/hexano com os demais solventes, sendo possível constatar a sua eficiência neste trabalho durante a extração de carotenoides para amostras de minitomate desidratado da variedade Sweet Grape. Ao se utilizar a combinação de acetona/hexano, os valores de absorbância foram maiores para os comprimentos de onda de interesse (450 nm e $470 \mathrm{~nm}$ ), seguido dos solventes acetona/éter de petróleo e acetona/clorofórmio, este último sendo o menos eficaz. Desse modo, o gráfico permitiu melhor visualização do comportamento dos solventes.

Silva (2001) avaliou a eficiência de solventes orgânicos na extração de carotenoides e, constatou que, para o tomate parcialmente desidratado, os solventes hexano-etanol (1:1), acetona e acetona-hexano (1:1)

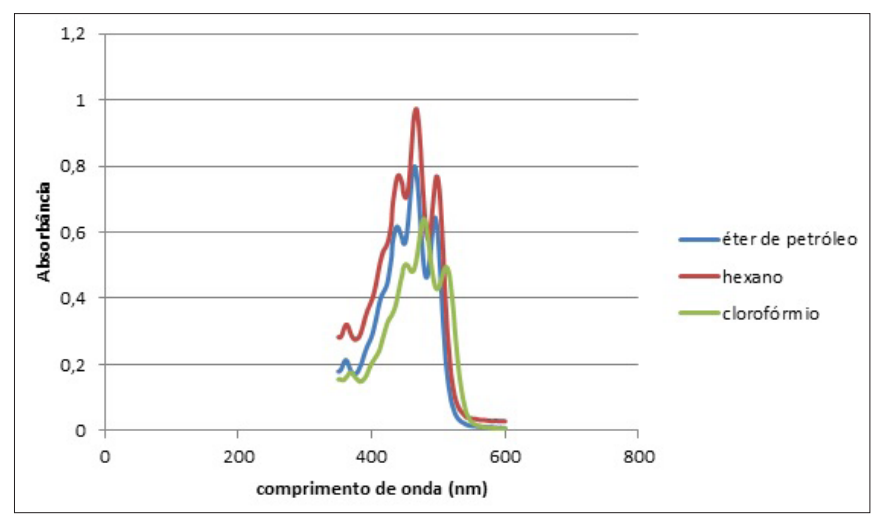

Figura 2. Espectro de UV/Vis de $350 \mathrm{~nm}$ a $600 \mathrm{~nm}$ das amostras de minitomate desidratado para diferentes solventes de extração. 
Extração e quantificação de carotenoides em minitomate desidratado (Sweet Grape) através da aplicação de diferentes solventes Nellis, S. C. et al.

apresentaram melhor poder de extração, corroborando com os resultados encontrados neste trabalho.

De acordo com Rodriguez-Amaya et al. (2008), fatores como a existência de um grande número de carotenoides, variabilidade qualitativa e quantitativa da composição dos alimentos, variação nas quantidades de carotenoides dentro de um mesmo alimento, distribuição não uniforme dos carotenoides entre amostras, a natureza variável das matrizes alimentícias, entre outros, são exemplos de fatores que tornam a análise de carotenoides dentro de um alimento muito difícil. Além disso, há fontes comuns de erros nessas análises, como: extração incompleta, perdas físicas ocorridas durante o procedimento, identificação equivocada, erros de quantificação ou cálculo, degradação oxidativa durante a análise ou estocagem da amostra, entre outros.

Neste trabalho, o objetivo principal foi o de qualificar o melhor solvente para extração dos carotenoides licopeno e beta-caroteno de minitomate desidratado da variedade Sweet Grape. Sendo assim, tais dados facilitam a tomada de decisão por parte do pesquisador, ao avaliar a combinação solvente e amostra, satisfazendo os propósitos de utilização, levando-se em consideração também o fator econômico e toxicidade dos solventes a serem utilizados.

\section{Conclusões}

Com base na pesquisa conduzida, conclui-se que o melhor solvente para extração de carotenoides em minitomate desidratado da variedade Sweet Grape, tanto para o pigmento licopeno como para o betacaroteno, foi a combinação de acetona como extrator inicial seguido do solvente hexano. Ao utilizá-los, foi possível quantificar uma concentração média de 25,71 e 26,53 mg $100 \mathrm{~g}^{-1}$ de betacaroteno e licopeno, respectivamente, nas amostras de minitomate, diferindo significativamente $(p<0,05)$ da extração com os demais solventes: acetona/clorofórmio e acetona/éter de petróleo.

Entretanto, ao se comparar os resultados com o de outros trabalhos, notou-se que a quantidade de carotenoides em determinado produto pode ser muito variável e que há erros comuns e fatores que dificultam estas análises, tais como a variação da concentração de carotenoides presente em um mesmo alimento ou até mesmo a existência de um grande número de carotenoides.

\section{Referências}

AGUIAR, F. P. C.; ABRAHÃO, R. M. S.; ANJOS, V. D. A. Determinação da vida útil de tomate tipo cereja e "Sweet Grape". In: CONGRESSO INTERINSTITUCIONAL DE INICIAÇÃO CIENTÍFICA, 7., 2012, Jaguariúna. Anais... Jaguariúna: CIIC, 2012. p. 56-60.

CARVALHO, P. G. B.; MACHADO, C. M. M.; MORETTI, C. L.; FONSECA, M. E. N. Hortaliças como alimentos funcionais. Horticultura Brasileira, Brasília, v. 24, n. 4, p. 147-152, 2006.

JUNQUEIRA, A. H.; PEETZ, M. S.; ONODA, S. M. Sweet Grape: um modelo de inovação na gestão da cadeia de produção e distribuição de hortaliças diferenciadas no Brasil. São Paulo: Central de Cases, 2011. 19 p. Disponível em: <http://www. organicsnet.com.br/wp-content/uploads/sweet_grape.pdf>

Acesso em: 13 ago. 2013.

LIMA, K. V. G.; PAIXÃO, J. A.; LUCENA, J. R. M.; OLIVEIRA, M. T.; TOMIYA, J. P. D. N. S. Carotenóides totais e licopeno em tomate, goiaba e acerola. Medicine, [S.I.], v. 227, p. 908-913, 2002.

MORAES, G. H. K.; BRUNE, W.; CAMBRAIA, J. Uma técnica para avaliação de $\alpha, \beta$-Caroteno e Licopeno em material vegetal. Revista Ceres, Viçosa, v. 21, n. 113, p. 63-72, 1974.

RODRIGUEZ-AMAYA, D. B. A guide to carotenoid analysis in foods. Washington: Internacional Life Sciences Institute Press, 2001. $64 \mathrm{p}$.

RODRIGUEZ-AMAYA, D. B.; KIMURA, M. Handbook for carotenoid analysis. Washington: HarvestPlus, 2004. 58 p. (HarvestPlus Technical Monograph, 2).

RODRIGUEZ-AMAYA, D. B.; KIMURA, M.; AMAYA-FARFAN, $J$. Fontes brasileiras de carotenoides: tabela brasileira de composição de carotenoides em alimentos. Brasília: Ministério do Meio Ambiente, Secretaria de Biodiversidade e Florestas, 2008.

SILVA, A.G. Extração e estabilidade dos carotenoides obtidos de tomate processado (Lycopersicon esculentum Mill). 2001. 142 f. Tese (Mestrado em Ciências)-Universidade Federal de Viçosa, Viçosa, 2001

STAHL, W.; SIES, H. Carotenoids: occurrence, biochemical activities, and bioavailability. In: PACKER, L.; HIRAMATSU, M.; YOSHIKAWA, T. Antioxidant food suplements in human health. San Diego: Academic Press, 1999. p. 183-198.

WILBERG, V. C.; RODRIGUEZ-AMAYA, D. B. Quantificação de $\beta$-caroteno e licopeno em tomate e em alguns dos seus produtos por cromatografia líquida de alta eficiência. Ciência e Tecnologia de Alimentos, Campinas, v. 13, n. 2, p. 132-141, 1993. 